\section{Severe fever with thrombocytopenia syndrome presenting as acute hepatic failure}

\author{
Jung Gil Park \\ Department of Internal Medicine, Gumi CHA Medical Center, CHA University, Gumi, Korea
}

Severe fever with thrombocytopenia syndrome (SFS) is characterized by fever, thrombocytopenia, leukopenia, and altered consciousness, which may also involve multi-organ failure. Initially SFTS mortality was as high as 30\%, when diagnosis remained unclear. We present a case of a 53year- old man with SFTS presenting with acute hepatic failure. On admission, he presented with confusion, elevated serum liver enzyme and ammonia levels, whose serum markers were negative for acute viral hepatitis. He was diagnosed with SFTS based on reverse transcription-polymerase chain reaction identification of the SFTS virus M segment. Percutaneous liver biopsy was performed to identify the degree and extent of necroinflammation and patient prognosis. After recovery, he was followed-up for 12 months with no SFTS-related sequelae. A discordance in severity between biopsy findings and clinical course could explain the rapid clinical improvement. Atypical presentations with multi-organ failure can delay timely diagnosis and management of infected patients.

Keywords Phlebovirus; Liver failure; Hepatitis; Bunyaviridae
eISSN: 2383-4625

Received: 18 February 2015

Revised: 12 March 2015

Accepted: 16 March 2015

Correspondence to: Jung Gil Park Department of Internal Medicine, Gumi CHA Medical Center, CHA University, 12 Sinsi-ro 10-gil, Gumi 730-728, Korea

E-mail: gsnrs@naver.com

\section{Capsule \\ Summary}

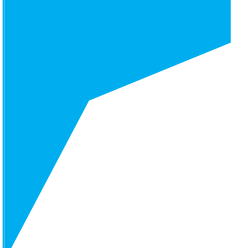

What is already known

Severe fever with thrombocytopenia syndrome is characterized by fever, thrombocytopenia, leukopenia, and altered consciousness involving multi-organ failure.

What is new in the current study

If a patient has acute hepatic failure secondary to systemic infection in an endemic area, severe fever with thrombocytopenia syndrome should be considered as the etiology.

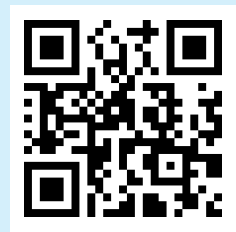

How to cite this article:

Park JG. Severe fever with thrombocytopenia syndrome presenting as acute hepatic failure. Clin Exp Emerg Med 2015;2(2):137-140.

This is an Open Access article distributed under the terms of the Creative Commons Attribution Non-Commercial License (http:// creativecommons.org/licenses/by-nc/3.0/). 


\section{INTRODUCTION}

Severe fever with thrombocytopenia syndrome (SFTS) is an emerging infectious disease first reported in China in 2010.' Epidemiologic data suggest its incidence is growing and expanding to other Asian and Western countries. ${ }^{2-4}$ Its etiology is the SFTS virus (SFTSV), grouped within the Bunyaviridae family and transmitted by the Haemaphysalis longicornis tick. ${ }^{1}$ Its peak incidence is from May to August in middle-aged residents of mountainous regions. SFTS is characterized by severe fever with thrombocytopenia, leukopenia, abdominal pain, nausea, and vomiting which can progress to multi-organ dysfunction and death in extreme cases; the case fatality has been reported to be as high as 30\%,5, Although its typical features can hint at SFIS diagnosis, associated organ failure can mask clinical characteristics, resulting in delayed diagnosis and treatment. We report a case of SFTS that presented as acute hepatic failure, and review clinical characteristics of SFTS in the literature. A male patient initially presented with altered consciousness and clinical and laboratory features of acute hepatitis. The patient was diagnosed with SFTS by serologic markers and polymerase chain reaction. SFTS should be considered as a cause of acute hepatitis in endemic areas during transmission seasons.

\section{CASE REPORT}

A 53-year-old man visited the emergency department for confusion that had started two days prior. He was a farmer in a rural area of South Korea. On admission, his body temperature was $37.3^{\circ} \mathrm{C}$, blood pressure was 115/85 mmHg, heart rate was 111 beats/min, and his respiration rate was 26 breaths/min. He appeared acutely ill with marked stupor and severe impairment in cognitive function, only responding to painful stimuli. Physical examination revealed a crusted ulcer with an erythematous rim on his right thigh. Laboratory tests showed a white blood cell count of $3.46 \times 10^{3} /$ $\mu \mathrm{L}$, hemoglobin level of $14.6 \mathrm{~g} / \mathrm{dL}$, platelet count of $55 \times 10^{3} / \mu \mathrm{L}$, aspartate aminotrasferase concentration of 3,404 IU/L, total bilirubin level of $1.61 \mathrm{mg} / \mathrm{dL}$, prothrombin time of 11.9 seconds, and ammonia level of $67 \mu \mathrm{mol} / \mathrm{L}$; he was also negative for all serum markers of acute viral hepatitis.

The clinical course after admission is shown in Fig. 1. On the third day, his consciousness deteriorated to a comatose state and mechanical ventilator therapy was initiated in the intensive care unit. Chest X-rays showed slightly increased vascular markings with increased cardiothoracic ratio (58\%). Transthoracic echocardiography revealed global hypokinesia of the left ventricle and moderately decreased ejection fraction (34\%). Serum troponin I

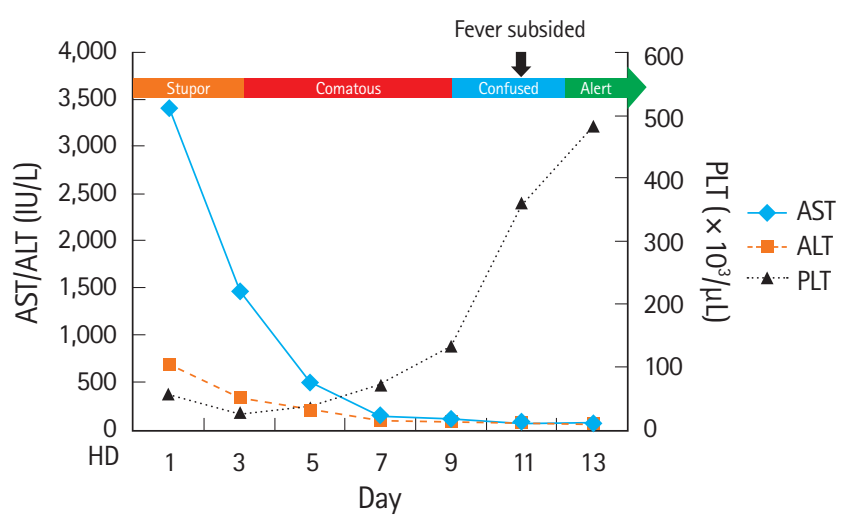

Fig. 1. Time course of fever and serum transaminase, platelet, and consciousness levels. AST, aspartate aminotransferase; ALT, alanine aminotransferase; PLT, platelet; HD, hostpital days.

and pro-brain natriuretic peptide levels were elevated $(0.768 \mathrm{ng} /$ $\mathrm{mL}$ and 2,480 pg/mL, respectively) without echocardiogram changes. On the sixth day of hospitalization, reverse transcription polymerase chain reaction performed at the Korea Center for Disease Control and Prevention (Cheongju, Korea) confirmed the SFTS diagnosis by identifying the M segment of the SFTSV. He remained comatose after successful weaning from mechanical ventilator therapy after five days. On day 9, a lumbar puncture was performed to confirm that there was no evidence of intracranial hypertension or infection of the central nervous system based on cerebrospinal fluid analysis (2 cells/ $/ \mathrm{mm}^{3}, 100 \%$ lymphocytes). Percutaneous liver biopsy was performed after platelet counts had rebounded to $215,000 / \mathrm{mm}^{3}$. Liver biopsy showed lymphocyte aggregation in the periportal area and the amount of inflammation was less extensive than expected based on biochemical parameters (Fig. 2). On day 11, the patient's mental status improved and his fever subsided below $38^{\circ} \mathrm{C}$. On day 14 , he had completely recovered motor power in all extremities. The ejection fraction of the left ventricle had recovered to $44 \%$ on the follow-up echocardiogram, and further increased to $55 \%$ on day 21 . There was no evidence of inducible ischemia on myocardial perfusion single photon emission computed tomography. The patient was followed up for 12 months after discharge with no SFTS-related sequelae.

\section{DISCUSSION}

SFTS is an emerging infectious disease caused by SFTSV, which belongs to a third distinct lineage within the Phlebovirus genus, a member of the Bunyaviridae family. The incidence rate of SFTS is growing and expanding in China. Moreover, outbreaks have also been reported in other Asian and Mediterranean countries as well as the United States. ${ }^{2-4}$ The incidence rate of SFTS varies from 0.03 to 0.05 per 1,000 based on province in China. ${ }^{7}$ SFT is diagnosed 

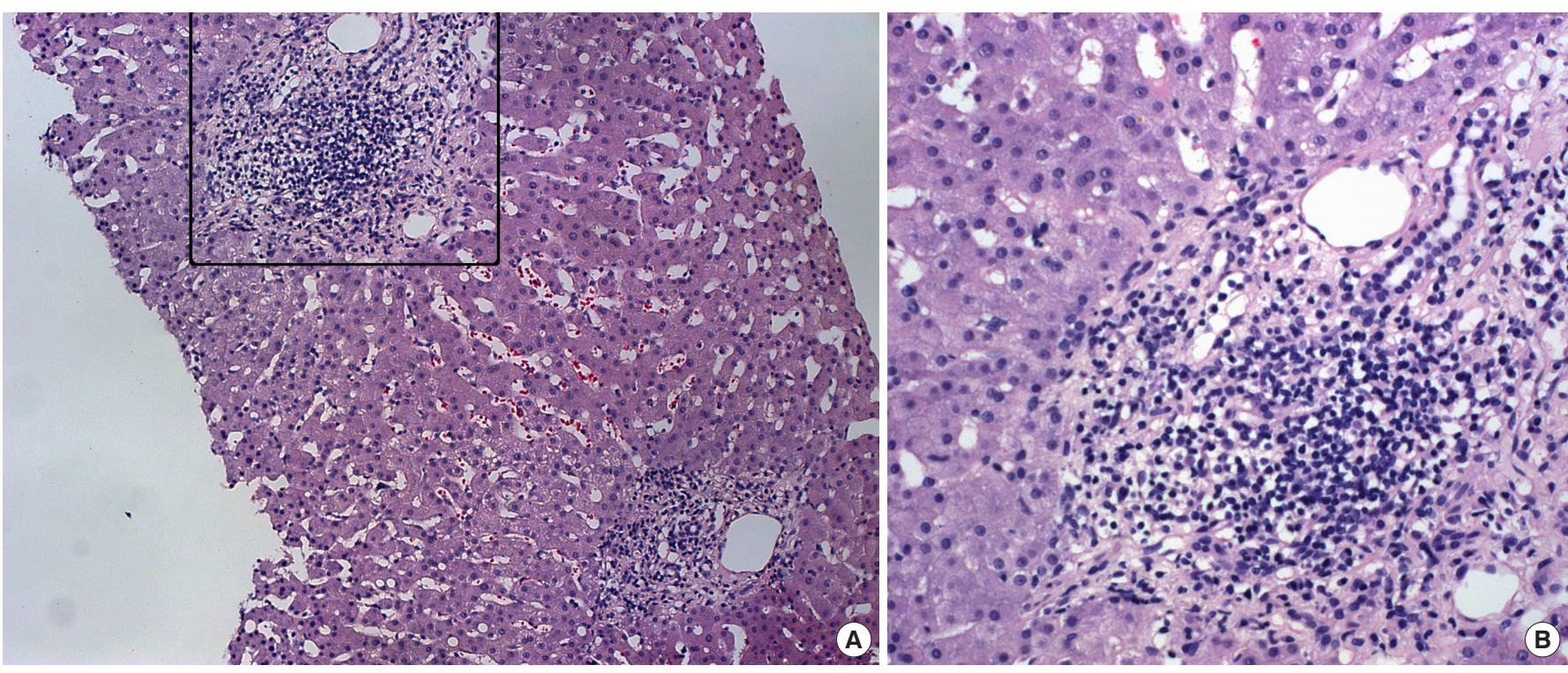

Fig. 2. Percutaneous liver biopsy showing lymphocyte aggregation with mild lobular activity in the periportal area. $H \& E_{1}(A) \times 100,(B) \times 200$.

based on viral nucleic acid or serological tests. Although the typical features of severe fever accompanied by thrombocytopenia in endemic areas can lead clinicians to suspect the virus, associated symptoms related to multi-organ failure may delay timely diagnosis by masking the characteristic features of SFTS. In this case, the patient presented with altered consciousness with marked elevation of serum aminotransferase and ammonia levels. After diagnosing him with acute hepatic failure associated with acute viral infection, we sought to identify the causative agent underlying the multi-organ failure.

The pathogenesis of SFT is not clearly understood because there is no established experimental animal model. In a murine C57BL/6 mice model of SFTSV infection, viral RNA was detected in the blood, spleen, liver, and kidney but not in the lungs, intestine, heart, muscle, or brain. The number of macrophages and platelets were greatly increased in the spleen, and SFTSV was detected in platelets isolated from the cytoplasm of macrophages. These findings suggest that thrombocytopenia might be attributable to clearance of circulating virus-infected platelets by macrophages in the spleen. ${ }^{8}$

The clinical course of SFTS is divided into fever, multi-organ dysfunction, and recovery stages. After an incubation period of 1 to 2 weeks, the fever stage is characterized by fever, flu-like symptoms, and laboratory abnormalities including thrombocytopenia, leukopenia, abnormal liver function test results, and elevated muscle enzyme levels. After a week, symptoms and muscle enzyme levels begin to resolve in survivors. In some patients, the disease progresses to the second stage, multi-organ dysfunction. In the third stage, nonfatal cases recover with normalization of labora- tory findings and clinical parameters. Fatal cases succumb to multiorgan failure, coagulopathy, shock, and acute respiratory distress syndrome. ${ }^{5,9}$

The standard SFT treatment is symptomatic and supportive therapy. ${ }^{7}$ However, plasma exchange with ribavirin might be used in patients with rapidly progressing SFTS. ${ }^{10}$ Though a vaccine for SFTSV is not yet available, repellents such as N,N-diethyl-m-toluamide or permethrin may protect against tick bites in endemic areas. ${ }^{11}$ In cases of direct exposure to blood of patients with SFI, oral ribavirin or human monoclonal antibodies can be used for disease prophylaxis.?

In the present case, acute viral infection was suggested as a cause of the acute hepatitis based on clinical parameters including fever, leukopenia, thrombocytopenia, and evidence of multiorgan involvement of the heart, muscle, kidneys, and lungs. The clinical course of the present case is well in accordance with the three standard phases of SFTS. The stage of fever with thrombocytopenia and leukopenia rapidly led to multi-organ dysfunction. Despite the severe laboratory abnormalities with multi-organ involvement, rapid normalization of platelet counts and serum aminotransferase levels might suggest better patient prognosis. Serum aminotransferase, creatine kinase, creatine kinase-MB, and lactate dehydrogenase levels in the multi-organ dysfunction stage of disease progression are reported to be major clinical indicators and risk factors associated with fatal outcome.

The patient was diagnosed with acute hepatic failure based on his altered mental status accompanied by marked deterioration in liver function test results. Computed tomography imaging of his brain did not show other obvious causes for his altered con- 
sciousness. His normal intracranial pressure and cerebrospinal fluid analysis excluded the possibility of direct viral involvement of the central nervous system. Therefore, there was no other obvious cause for his mental status change except hepatic encephalopathy with acute deterioration of liver function and elevated serum ammonia levels.

We performed liver biopsy to identify the degree and extent of necroinflammation and the prognosis during the recovery phase, when platelet counts had returned to normal levels. A discordance in severity between biopsy findings and clinical course may explain rapidly reduced inflammation and virus clearance during the recovery phase in nonfatal patients. To our knowledge, this is the first reported case of a patient with SFTS who received a liver biopsy.

In conclusion, we report a case of a patient who presented with acute hepatic failure secondary to systemic SFISV infection. SFTS should be considered for patients in endemic areas with severe fever with thrombocytopenia and multi-organ dysfunction. Prompt SFTS recognition and diagnosis can allow timely treatment and predict prognosis of patients with severe multi-organ failure.

\section{CONFLICT OF INTEREST}

No potential conflict of interest relevant to this article was reported.

\section{REFERENCES}

1. Yu XJ, Liang MF, Zhang SY, et al. Fever with thrombocytopenia associated with a novel bunyavirus in China. N Engl J Med 2011;364:1523-32.

2. Denic S, Janbeih J, Nair S, Conca W, Tariq WU, Al-Salam S. Acute thrombocytopenia, leucopenia, and multiorgan dysfunction: the first case of SFTS bunyavirus outside China? Case Rep Infect Dis 2011;2011:204056.

3. Charrel RN, Moureau G, Temmam S, et al. Massilia virus, a novel Phlebovirus (Bunyaviridae) isolated from sandflies in the Mediterranean. Vector Borne Zoonotic Dis 2009;9:51930.

4. McMullan LK, Folk SM, Kelly AJ, et al. A new phlebovirus associated with severe febrile illness in Missouri. N Engl J Med 2012;367:834-41.

5. Gai $Z$, Liang $M$, Zhang $Y$, et al. Person-to-person transmission of severe fever with thrombocytopenia syndrome bunyavirus through blood contact. Clin Infect Dis 2012;54:249-52.

6. Chang MS, Woo JH. Severe fever with thrombocytopenia syndrome: tick-mediated viral disease. J Korean Med Sci 2013; 28:795-6.

7. Liu Q, He B, Huang SY, Wei F, Zhu XO. Severe fever with thrombocytopenia syndrome, an emerging tick-borne zoonosis. Lancet Infect Dis 2014;14:763-72.

8. Jin $C$, Liang $M$, Ning J, et al. Pathogenesis of emerging severe fever with thrombocytopenia syndrome virus in C57/BL6 mouse model. Proc Natl Acad Sci U S A 2012;109:10053-8.

9. Li D. A highly pathogenic new bunyavirus emerged in China. Emerg Microbes Infect 2013;2:e1.

10. Oh WS, Heo ST, Kim SH, et al. Plasma exchange and ribavirin for rapidly progressive severe fever with thrombocytopenia syndrome. Int J Infect Dis 2014;18:84-6.

11. Cisak E, Wojcik-Fatla A, Zajac V, Dutkiewicz J. Repellents and acaricides as personal protection measures in the prevention of tick-borne diseases. Ann Agric Environ Med 2012;19:62530. 